\title{
Do gene-environment interactions play a role in COVID-19 distribution? The case of Alpha-1 Antitrypsin, air pollution and COVID-19
}

\author{
Nicola Murgia, ${ }^{1}$ Angelo Guido Corsico, ${ }^{2}$ Gennaro D'Amato, ${ }^{3}$ Cara Nichole Maesano, ${ }^{4}$ Arturo Tozzi,${ }^{5}$ \\ Isabella Annesi-Maesano ${ }^{4,6}$ \\ ${ }^{I}$ Section of Occupational Medicine, Respiratory Diseases and Toxicology, University of Perugia, Italy \\ ${ }^{2}$ Center for Diagnosis of Inherited a1-Antitrypsin Deficiency, Department of Internal Medicine and Therapeutics, \\ Pneumology Unit, Fondazione IRCCS Policlinico San Matteo, University of Pavia, Italy \\ ${ }^{3}$ Division of Respiratory and Allergic Diseases, Department of Chest Diseases, High Specialty A. Cardarelli Hospital, and \\ Medical School of Specialization in Respiratory Diseases, Federico II University of Naples, Italy \\ ${ }^{4}$ INSERM and Sorbonne University, Epidemiology of Allergic and Respiratory Diseases Department, IPLESP, Paris, France \\ ${ }^{5}$ Center for Nonlinear Science, Department of Physics, University of North Texas, Denton TX, USA \\ ${ }^{6}$ Desbrest Institute of Epidemiology and Public Health, INSERM and Montpellier University, Montpellier, France
}

\begin{abstract}
Background: Gene-environment interactions are relevant for several respiratory diseases. This communication raises the hypothesis that the severity of COVID-19, a complex disease where the individual response to the infection may play a significant role, could partly result from a gene-environment interaction between air-pollution and Alpha-1 Antitrypsin (AAT) genes.

Methods: To evaluate the impact of the AAT and air pollution interaction on COVID-19, we introduced an AAT*air pollution global risk score summing together, in each country, an air pollution score (ozone, nitrogen dioxide and fine particulate matter) and an AAT score (which sums the ranked frequency of MZ, SZ, MS). We compared this global score with the ranking of European countries in terms of death number per million persons.

Results: The ranking of the AAT*air pollution global risk score matched the ranking of the countries in terms of the observed COVID-19 deaths per 1M inhabitants, namely in the case of the first European countries: Belgium, UK, Spain, Italy, Sweden, France. We observed parallelism between the number of COVID deaths and the AAT*air pollution global risk in Europe. AAT anti-protease, immune-modulating and coagulation-modulating activities may explain this finding, although very speculatively.

Conclusions: Even if further studies taking into account genetic background, population density, temporal dynamics of individual epidemics, access to healthcare, social disparities and immunological response to SARS-CoV2 are needed, our preliminary observation urges to open a discussion on gene-environment interactions in COVID-19.
\end{abstract}

Key words: AAT; COVID-19; environment; air pollution.

Correspondence: Nicola Murgia, Section of Occupational Medicine, Respiratory Diseases and Toxicology, University of Perugia, Piazzale Gambuli, 06128 Perugia, Italy. Tel. +39.075.5784488. E-mail: nicola.murgia@unipg.it

Contributions: NM, conceptualization, investigation, original draft writing; AGC, AT, validation, manuscript review and editing; GDA, conceptualization, manuscript review and editing; CNM, methodology, manuscript review and editing; IAM, conceptualization, formal analysis, supervision, original draft writing.

Conflict of interest: The authors declare that they have no competing interests, and all authors confirm accuracy. IAM is an Associate Editor of Multidisciplinary Respiratory Medicine, GDA is a member of the Editorial Board of Multidisciplinary Respiratory Medicine.

Availability of data and materials: Available from the corresponding author on reasonable request.

Ethics approval and consent to participate: Not applicable.

Consent for publication: Not applicable. 


\section{Introduction}

Gene-environment interactions are thought to be critical for several respiratory diseases such as cancer, emphysema and asthma. They could contribute also to the respiratory infection caused by SARS-CoV-2 that deeply affects the lung by increasing the risk of suffering from a severe form of the disease (COVID-19) in susceptible individuals. Genetic susceptibility to SARS-CoV2 has been studied: some critical immunogenetic pathways (e.g., interferon related) have been identified and interconnection with other important system (e.g., renine-angiotensin-aldosterone) in infection progression has been proposed [1]. Another relevant factor in the pathogenesis of COVID-19 seems to be Alpha-1 Antitrypsin (AAT) which shows an inhibitory effect on transmembrane serine protease 2 (TMPRSS2), key protease for SARS-CoV2 infection [2]. AAT deficiency (AATD) is a genetic condition resulting from the inheritance of two abnormal alpha-1 (Z) antitrypsin genes (ZZ) impacting AAT production, a protein which protects the lungs from inflammation caused by infection and inhaled irritants. Individuals with the AAT genetic defect do not release alpha-1-antiprotease from the liver, and present low levels of the protein in serum and alveoli. Consequently, alveoli lack antiprotease protection from external aggressions. Although this deficiency is rare, other more frequent AAT gene combinations exist (SZ, SS, MS, MZ among the commonest ones) that also modulate AAT production [3]. Gene involvement in COVID-19 is supported by the fact that ethnic differences in AATD alleles might explain national differences in COVD-19 fatality, although only partly [4].

For some authors, air pollution may play a role in SARS-CoV2 infection and COVID-19 severity [5]. However, it is not clear yet if airborne pollutants are directly involved in causing a more severe form of the disease or are the chronic diseases correlated to air pollution (cardiovascular and respiratory) to make the subject more prone to the detrimental effect of the infection [6].

In this short report we raise the hypothesis according to which the severity of COVID-19 could partly result from a gene-environment interaction between air pollution and AAT genes that impair lung and immunological response to SARS-CoV-2 action and boost an exaggerated inflammatory response, responsible of COVID-19 lung damage.

\section{Methods}

To explore the impact of the AAT and air pollution interaction on COVID-19, we consider the situation in Europe, where both AAT Deficiency has been mapped and quantified and air pollution exposure data are available. Similar data are not available in the rest of the world.

AAT Deficiency frequencies according to major genotypes was drawn by the results of an international study [3] and air pollution level by the European Environmental Agency (https://www.eea.europa.eu/themes/air). COVID-19 death numbers were provided by https://coronavirus.jhu.edu/map.html.

To evaluate the impact of the AAT and air pollution interaction on COVID-19, we introduced a $\mathrm{AAT}_{*}$ air pollution global risk score summing together, in each country, an air pollution score (which sums exposure levels (low $=1$, medium $=2$, high=3) for each of three major pollutants (ozone, nitrogen dioxide and fine particulate matter) and an AAT score (which sums the ranked frequency of MZ, SZ, MS deficits according to five classes from absent $=1$ to elevated=5). We compared this global score with the ranking of countries in terms of death number per million persons.

\section{Results}

The ranking of the $\mathrm{AAT}_{*}$ air pollution global risk score matched the ranking of the countries in terms of the observed COVID-19 deaths per $1 \mathrm{M}$ inhabitants, namely in the case of the first European countries: Belgium (1385), Spain (949), Italy (875), UK (838), France (780) (Figure 1).

\section{Discussion}

We observed an ecological parallelism between the number of COVID-19 deaths and the AAT $*$ air pollution global risk in Europe.

This observation is supported by biological plausibility. As a matter of fact there is an increasing evidence of the role of inflammation/AAT imbalance in making COVID-19 more severe [7] which is influenced by air pollution: AAT has an inhibitory effect on important infection and disease mediators, such as ADAM-17 [2], and down-regulate the production of IL-6, IL-8, IL-1b, and TNF-a, key mediators in the "cytokine storm" observed in severe COVID-19 pneumonia patients and possibly related to a macrophage activation syndrome and a secondary haemophagocytic lymphohistiocytosis [8]. Pneumothorax and pneumomediastinum are well-known complications of ventilated COVID-19 patients, explained by the use of higher pressures and larger tracheal tubes, but also by diffuse alveolar damage which may contribute to air leakage. It is unclear if antiproteases play a protective role in SARS-CoV-2 alveolar damage, but AAT deficiency has previously been identified as a predisposing factor for pneumothorax [9]. Systemic thrombosis could be another important mechanism of lung and systemic damage in COVID-19 patients. In a recent survey, patients with severe AAT deficiency had a higher mortality from pulmonary embolism than the reference population [10]. This finding has not been explained, but may be related to an increased complement activation, as evidenced by patients with AAT deficiency who show an increased cleavage of complement $\mathrm{C} 3$ component in $\mathrm{C} 3 \mathrm{~d}$ [11]. Moreover, the crosstalk between complement, coagulation and the vascular system is actually considered one of the most important factors in determining coagulation abnormalities and thrombosis seen in the more severe COVID-19 patients [12]. Complement system can be activated by virus-IgG or virusIgM immunocomplexes or by virus-IgA immunocomplexes, through mannose-binding lectin linked to both viral N-glycan and $\operatorname{IgA}[13]$.

One concern about our findings may raise up from the observation of a very low prevalence of COPD, which is one typical clinical outcome in AAT-deficiency, in patients hospitalized for COVID-19. However, COPD was strongly associated with the more severe forms of COVID-19, requiring mechanical ventilation and ICU admission [14].

The main limitation of our observation is that many other factors could explain the overlap between environment and COVID-19. One of the most important is population density, which could contribute substantially to environmental pollution. However there is increasing evidence of a role of air pollution in COVID-19. mortality taking into account also population density, as well as other important factors such as: family size, house crowding, percentage of private mobility use, and socio-economical deprivation [15].

Large genetic studies on the interaction between the human genome and SARS-CoV-2 infection are already ongoing (for example https://www.covid19hg.org/), but they do not explain COVID-19 severity entirely and not consider environmental interactions. Further studies considering population density, temporal 


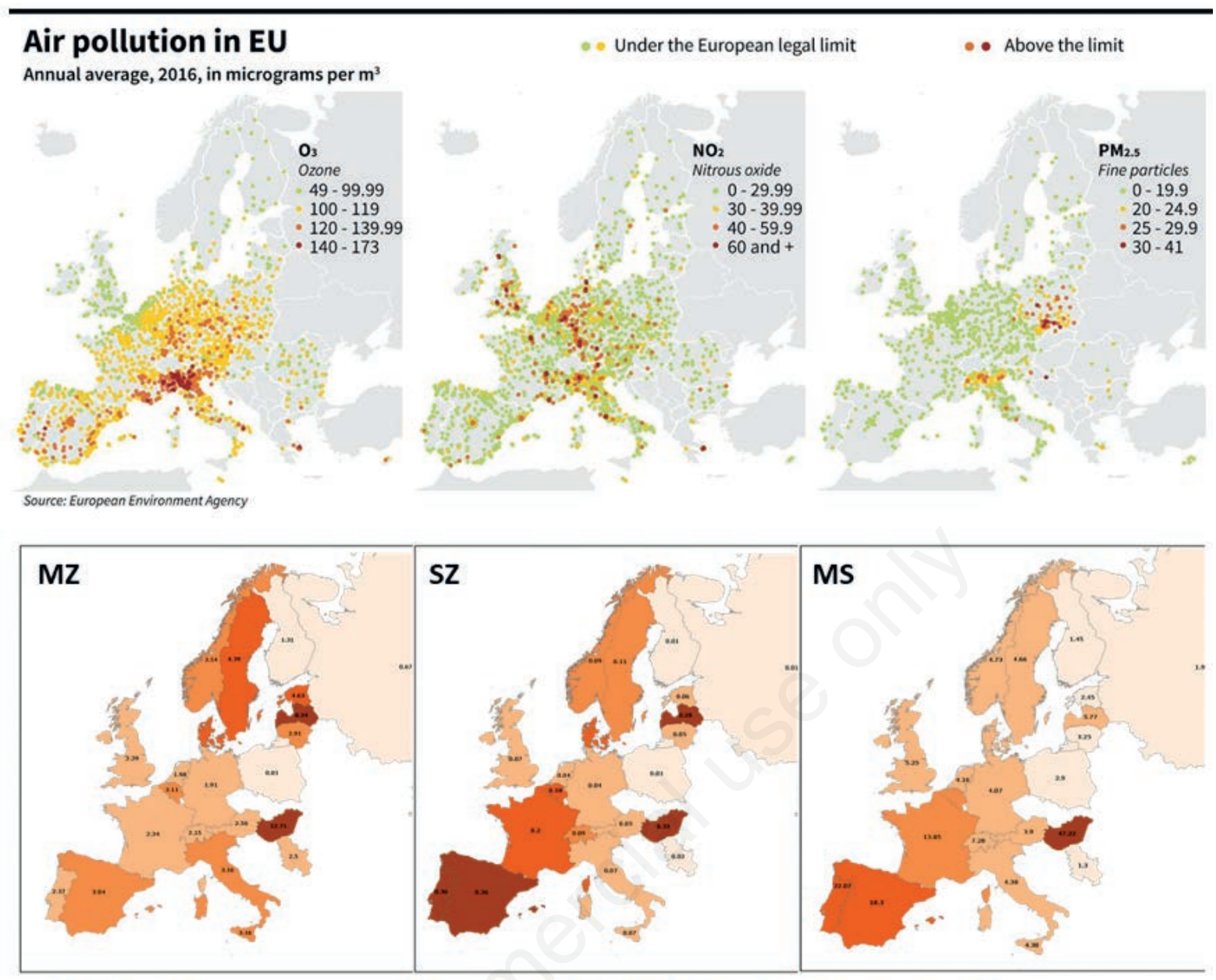

AAT deficient genes combination (MS, SZ, MS) distribution in EU

Figure 1. Air pollution and alpha-1 antitrypsin (ATT) genes combinations distribution in EU.

dynamics of individual epidemics, access to healthcare, social disparities and immunological response to SARS-CoV-2 are needed. Adhering to the precautionary principle, however, suggests air pollution emissions must be diminished now to protect individuals.

\section{Conclusions}

Our observation, though very preliminary, should help to open a discussion on gene-environment interactions in COVID-19. Moreover, even if most of the research is still ongoing, respiratory doctors and other specialists should be aware of emerging clinical predisposing factors and their interactions with host immunity and coagulation, which would make more susceptible the patients to the consequences of SARS-CoV-2 infection.

\section{References}

1. McCoy K, Peterson A, Tian Y, Sang Y. Immunogenetic association underlying severe COVID-19. Vaccines (Basel)
2020;8:E700.

2. de Loyola MB, Dos Reis TTA, de Oliveira GXLM, da Fonseca Palmeira J, Argañaraz GA, Argañaraz ER. Alpha-1-antitrypsin: A possible host protective factor against Covid-19. Rev Med Virol 2020:e2157.

3. Blanco I, de Serres FJ, Fernandez-Bustillo E, Lara B, Miravitlles M. Estimated numbers and prevalence of $\mathrm{PI} * \mathrm{~S}$ and $\mathrm{PI}{ }^{*} \mathrm{Z}$ alleles of alpha1-antitrypsin deficiency in European countries. Eur Respir J 2006;27:77-84.

4. Shapira G, Shomron N, Gurwitz D. Ethnic differences in alpha-1 antitrypsin deficiency allele frequencies may partially explain national differences in COVID-19 fatality rates. FASEB J 2020;34:14160-5.

5. Pozzer A, Dominici F, Haines A, Witt C, Münzel T, Lelieveld J. Regional and global contributions of air pollution to risk of death from COVID-19. Cardiovasc Res 2020;116:2247-53.

6. Barouki R, Kogevinas M, Audouze K, Belesova K, Bergman A, Birnbaum L, et al. The COVID-19 pandemic and global environmental change: Emerging research needs. Environ Int 2020;146:106272.

7. McElvaney OJ, McEvoy NL, McElvaney OF, Carroll TP, Murphy MP, Dunlea DMP, et al. Characterization of the 
inflammatory response to severe COVID-19 illness. Am J Respir Crit Care Med 2020;202:812-21.

8. Gonagle D, Sharif K, O'Regan A, Bridgewood C. The role of cytokines including interleukin-6 in COVID-19 induced pneumonia and macrophage activation syndrome-like disease. Autoimmun Rev 2020;19:102537.

9. Boone PM, Scott RM, Marciniak SJ, Henske EP, Raby BA. The genetics of pneumothorax. Am J Respir Crit Care Med 2019;199:1344-57.

10. Tanash HA, Ekström M, Wagner P, Piitulainen E. Cause-specific mortality in individuals with severe alpha 1-antitrypsin deficiency in comparison with the general population in Sweden. Int J Chron Obstruct Pulmon Dis 2016;11:1663-9.

11. O'Brien ME, Fee L, Browne N, Carroll TP, Meleady P, Henry M. Activation of complement component 3 is associated with airways disease and pulmonary emphysema in alpha-1 antitrypsin deficiency. Thorax 2020;75:321-30.
12. Cugno M, Meroni PL, Gualtierotti R, Griffini S, Grovetti E, Torri A, et al. Complement activation in patients with COVID19: A novel therapeutic target. J Allergy Clin Immunol 2020;146:215-7.

13. Matricardi PM, Dal Negro RW, Nisini R. The first, holistic immunological model of COVID-19: Implications for prevention, diagnosis, and public health measures. Pediatr Allergy Immunol 2020;31:454-70.

14. Lippi G, Henry BM. Chronic obstructive pulmonary disease is associated with severe coronavirus disease 2019 (COVID-19). Respir Med 2020;167:105941.

15. De Angelis E, Renzetti S, Volta M, Donato F, Calza S, Placidi D, et al. COVID-19 incidence and mortality in Lombardy, Italy: an ecological study on the role of air pollution, meteorological factors, demographic and socioeconomic variables. Environ Res 2021;195:110777. 